\title{
Mathematical model of the fluid status after intravenous fluid infusion
}

Tilaï T. Rosalina, Peter H.M. Bovendeerd

Dept. of Biomedical Engineering, Eindhoven University of Technology

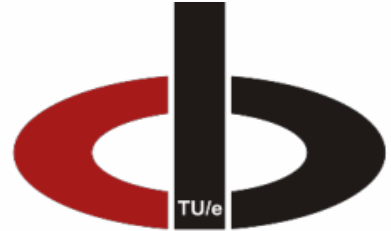

cardiovascular biomechanics

\section{Introduction}

Finding the optimal fluid balance in surgical patients is complex due to the lack of information on circulatory and interstitial volume through direct measurements.

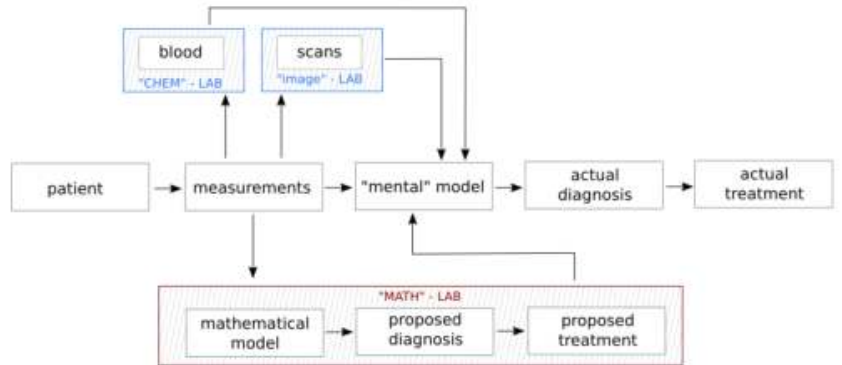

Figure 1: Clinical decision support workflow, with the addition of a mathematical laboratory

Mathematical models describing underlying physics and physiology, might be able to assist in determining the optimal fluid administration protocol for individual patients, by translating available measurements into quantitative information on the fluid status (figure 1).

\section{Aim}

In this study we present and test a pilot model for clinical decision support in fluid administration.

\section{Methods}

The model (figure 2) combines a cycle-averaged cardiovascular module including baro reflex control, with a fluid exchange module, driven by hydrostatic and osmotic pressure as described by Starling 2,3 . Urine flow is linearly related to plasma volume.

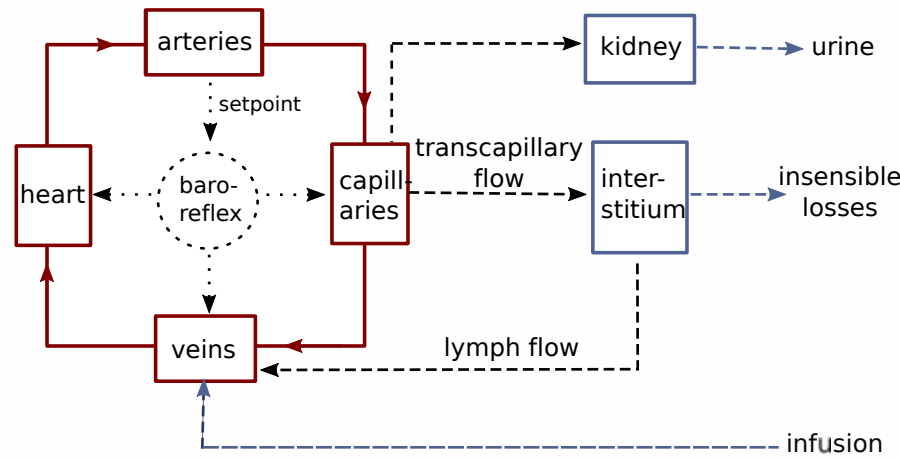

Figure 2: Schematic overview of the mathematical model
We fitted and tested the model by simulating an experiment in which $2.3 \mathrm{~L}$ of saline was infused over 23 minutes in volunteers ${ }^{4}$.

\section{Results}

In agreement with the experimental data, in the model initially (30-60 min) a substantial part of the infused fluid enters the interstitial space, and eventually (60-180 $\mathrm{min}$ ) leaves the body as urine.
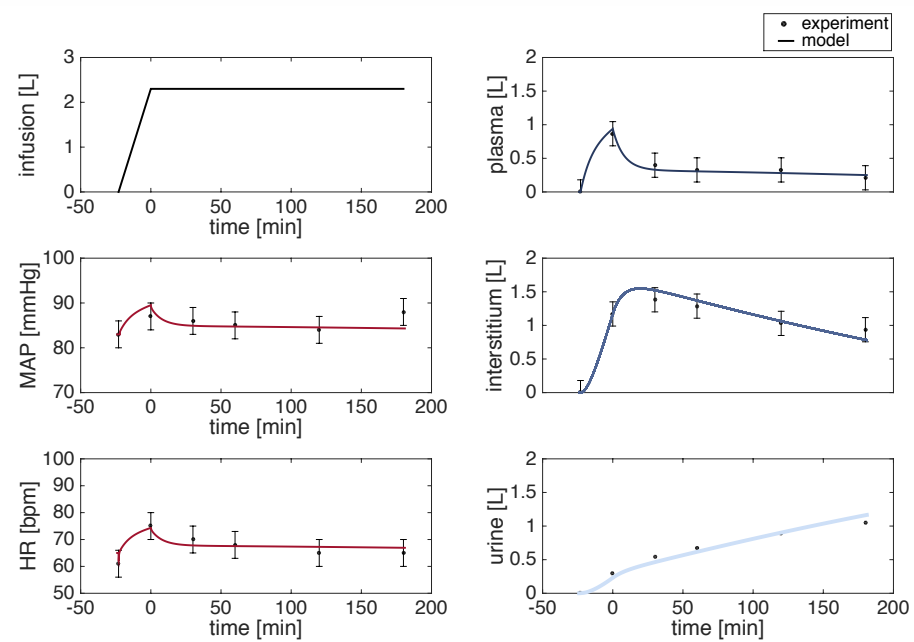

Figure 3: Experimental data ${ }^{4}$ compared to model results

\section{Discussion}

This general fluid exchange model is able to qualitatively capture the physiological changes in healthy volunteers after fluid infusion.

\section{Outlook}

This is the first step in exploring the potential of mathematical decision support tools for fluid administration. Next steps include performing a sensitivity analysis to find unique solutions, modelling the effect of anesthesia, and testing on different patient datasets.

\section{Acknowledgements}

This work was performed within the IMPULS II perioperative monitoring framework

\section{References \\ 1. Brandstrup et al. (2005) \\ 2. Jongen et al. (2016)}

3. Xie et al. (1995)

4. Watenpaugh et al. (1992) 